\title{
CORRECTION
}

\section{Correction to: Sulodexide in the Treatment of Chronic Venous Insufficiency: Results of the All-Russian Multicenter ACVEDUCT Program}

\author{
Andrey V. Chupin - Sergey E. Katorkin · Ivan I. Katelnitsky • \\ Oksana V. Katelnitskaya - Igor I. Prostov - Alexey S. Petrikov · \\ Alexander P. Koshevoi · Larisa F. Lyudkova
}

Published online: August 30, 2020

(c) Springer Healthcare Ltd., part of Springer Nature 2020

Correction to: Adv Ther (2020) 37:2071-2082

https://doi.org/10.1007/s12325-020-01270-9

In the original article, the "Duration, Types of Therapy and Dynamics of Improvements" topic published incorrectly.

The correct version is "The number of doctor visits for patients according to the protocol ranged from 3 to 5 (mean \pm SD $3.6 \pm 0.8$ visits). The mean \pm SD duration of sulodexide therapy

The original article can be found online at https://doi. org/10.1007/s12325-020-01270-9.

A. V. Chupin $(\square)$

Federal Scientific Clinical Centre of the Federal

Medical and Biological Agency of Russia, Moscow, Russia

e-mail: achupin@rambler.ru

S. E. Katorkin

Samara State Medical University of the RF Ministry of Public Health, Samara, Russia

I. I. Katelnitsky · O. V. Katelnitskaya · I. I. Prostov Rostov State Medical University of the RF Ministry of Public Health, Rostov, Russia

A. S. Petrikov

Altai State Medical University of the RF Ministry of Public Health, Barnaul, Russia

A. P. Koshevoi

Clinic "SibMedCentre", Tomsk, Russia

L. F. Lyudkova

Krasnoyarsk Interregional Polyclinic No 1,

Krasnoyarsk, Russia was $46.8 \pm 13.1$ days. The duration of therapy increased with CEAP class, from $29.9 \pm 14.5$ days in patients with $\mathrm{C} 1$ to $48.9 \pm 13.8$ days in those with C6. The majority of patients $(63 \% ; n=1417)$ visited the doctor three times and used parenteral and oral formulations of sulodexide $(85 \% ; n=1203)$ during the treatment period. Of the remaining 214 patients, $170(71 \%)$ only received sulodexide orally and 44 (29\%) only received the drug parenterally". 